\title{
Emotions et cognition : stratégie pour répondre à la question de la sensibilité des animaux
}

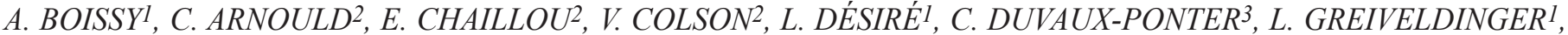 \\ C. LETERRIER ${ }^{2}$, S. RICHARD ${ }^{2}$, S. ROUSSEL ${ }^{3}$, H. SAINT-DIZIER ${ }^{2}$, M.C. MEUNIER-SALAÜN ${ }^{4}$, D. VALANCE ${ }^{2}$ \\ ${ }^{1}$ INRA, UR1213 Herbivores, F-63122 Saint-Genès Champanelle, France \\ 2 INRA, CNRS, Université de Tours, Haras Nationaux, UMR85 Physiologie de la Reproduction et des Comportements, \\ F-37380 Nouzilly, France \\ 3 INRA, INA-PG, UMR791 Physiologie de la Nutrition et de l'Alimentation, F-75231 Paris, France \\ ${ }^{4}$ INRA, Agrocampus, UMR1079 Systèmes d'Elevage, Nutrition Animale et Humaine, F-35590 Saint-Gilles, France
}

Courriel : alain.boissy@clermont.inra.fr

La question du bien-être de l'animal en élevage ne se pose que si l'on reconnaît à celui-ci le statut d'être sensible et réactif. Cela revient à considérer que l'animal est capable d'émotions, cherchant à minimiser les émotions négatives (peur, frustration...) tout en recherchant les émotions positives (plaisir, joie...) (Dawkins 1983, Duncan 1996). En plus des émotions qui sont par définition fugaces, le bien-être implique l'existence d'états affectifs persistants qui orientent la manière dont l'individu perçoit et réagit à son environnement (Lazarus 1991). Cependant, comme le soulignent Dawkins (2001) et Dantzer (2002), la recherche sur le bien-être des animaux d'élevage s'est généralement limitée à établir des indicateurs de stress sans pour autant pouvoir les relier à l'existence d'états affectifs. Il est désormais nécessaire de passer de la simple description des comportements de l'animal à la compréhension de ses propres états émotionnels (Désiré et al 2002, Mendl et Paul 2004).

Classiquement, une émotion se définit au travers d'une composante subjective qui est l'expérience émotionnelle proprement dite, et de deux composantes expressives, l'une motrice et l'autre physiologique (Dantzer 1989). La composante subjective est généralement estimée chez l'Homme à partir de ses rapports verbaux. Par contre chez l'animal, elle est inférée uniquement à partir des composantes motrice et physiologique. La nature exacte de ses émotions reste donc mal connue, et l'existence d'une communauté subjective entre l'Homme et les autres animaux est difficilement acceptée par la communauté scientifique. Les animaux sont pourtant capables d'attribuer une valeur affective à leur environnement. Les études sur le stress montrent que c'est la manière dont l'animal se représente l'événement, et non l'événement en tant que tel, qui va déterminer sa réaction : par exemple, ce n'est pas tant l'absence de nourriture mais plutôt la perception d'une privation qui est à l'origine du stress (Mason 1971). D'où la nécessité de prendre en compte les processus cognitifs, i.e. traitement de l'information et représentation mentale, pour parvenir à mieux appréhender l'expérience émotionnelle ressentie par l'animal. Les théories de l'évaluation développées en psychologie cognitive (Scherer 1999) offrent un cadre conceptuel qui peut être au moins en partie transposé à l'animal puisqu'il y est fait abstraction de la communication verbale et qu'elles sont basées sur des processus cognitifs élémentaires. Selon Scherer (1987), l'émotion naît de l'évaluation de l'événement par l'individu à partir d'un nombre limité de critères élémentaires (soudaineté, familiarité, prévisibilité et agrément de l'événement, correspondance aux attentes de l'individu, et possibilités de contrôle) ; la nature même de l'émotion dépend alors de la combinaison des critères d'évaluation.

Ce texte fait une rapide revue des interactions entre émotions et cognition, dont l'étude plus approfondie chez les animaux en production devrait permettre une meilleure compréhension de leurs états de bien-être. Une première partie traite des processus cognitifs impliqués dans le déclenchement et la différenciation des émotions. Une seconde partie, plus prospective, s'intéresse aux effets en retour des émotions sur les processus cognitifs, en explorant plus particulièrement le rôle de filtre plus ou moins persistant que jouent les émotions dans la manière dont l'animal se représente les événements auxquels il est exposé.

\section{1 / De la cognition dans les émotions ou comment accé- der au vécu émotionnel de l'animal}

Après avoir défini un cadre conceptuel inspiré des travaux en psychologie cognitive, nous avons engagé une approche originale des émotions chez les animaux d'élevage (ovin, caprin, porcin, caille) qui est basée sur leurs capacités cognitives. Concrètement, l'approche consiste à rechercher si les critères élémentaires d'évaluation, tels qu'ils sont identifiés chez l'Homme (soudaineté, familiarité,...), sont perçus par les animaux et s'ils donnent lieu à des états émotionnels reconnaissables par le biais de modifications comportementales et physiologiques (Désiré et al 2002). Le développement préalable d'une analyse fine des comportements (posture de la tête, des oreilles...) a été nécessaire. De même, des techniques non invasives (analyse de la variabilité de la fréquence cardiaque) ont dû être mises au point pour mesurer l'activation du système neurovégétatif à l'origine des modifications physiologiques (ovin : Després et al 2003, caille : Valance et al 2007b).

Des situations expérimentales, destinées à orienter le traitement cognitif des animaux, ont été élaborées pour isoler les critères élémentaires d'évaluation qui leur sont accessibles, tels 
Figure 1. Mesure de la période cardiaque (inverse de la fréquence cardiaque) d'agnelles exposées à un objet familier ou inconnu et présenté lentement ou rapidement.

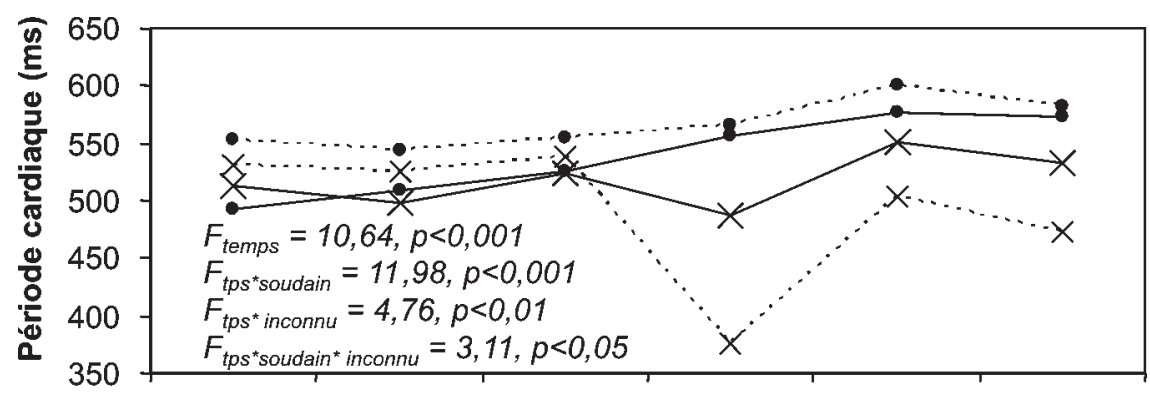

Temps calculé par rapport à la présentation du stimulus (s)

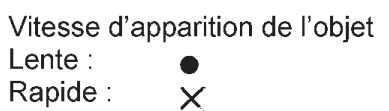

Type d'objet

Familier :

Inconnu:

La diminution de la période cardiaque (autrement dit l'accélération cardiaque) spécifiquement induite par la soudaineté est exacerbée lorsque l'événement soudain est également inconnu.

que la soudaineté et la non-familiarité de la situation. Des réponses émotionnelles propres à chacun de ces deux critères ont pu être définies : la présentation soudaine d'un objet provoque un sursaut et une brève accélération cardiaque alors que la présentation d'un objet non familier déclenche un comportement d'orientation vers l'objet et une augmentation transitoire de la variabilité de sa fréquence cardiaque (Désiré et al 2004, Roussel et al 2005, Valance et al 2007a). La combinaison de ces deux critères a un effet synergique sur les réponses émotionnelles de l'animal : l'accélération cardiaque spécifique à la soudaineté est accentuée dans le cas où l'événement soudain est également inconnu (Désiré et al 2006, figure 1). Les réponses émotionnelles à la soudaineté (sursaut et tachycardie) sont réduites lorsque l'animal a la pos- sibilité de prévoir l'événement déclencheur (Greiveldinger et al 2006, figure 2). De même, l'animal est capable d'élaborer des attentes, et l'écart entre ses propres attentes et la situation déclenche une agitation comportementale et une accélération cardiaque. Ces premiers résultats montrent clairement que les critères d'évaluation définis chez l'Homme pour évaluer la charge émotionnelle d'une situation donnée peuvent être communs à ceux utilisés par les animaux.

Dans une orientation plus finalisée, nous nous sommes intéressés aux rôles que pouvaient jouer les congénères dans le déclenchement des émotions. Les animaux sont fréquemment exposés à des modifications de leur environnement social. Par exemple, les réallotements impliquent généralement une rupture des liens avec les partenaires familiers et une confrontation avec des congénères inconnus, et les traitements sanitaires nécessitent souvent un isolement. Or, la confrontation d'animaux inconnus ou le simple isolement est à l'origine de réactions de stress très marquées chez les espèces domestiques (Boissy et al 2001a). Nous avons alors comparé entre mammifères (ovins, caprins et porcins) et oiseaux (cailles) les conséquences émotionnelles induites par la modification ponctuelle ou durable de l'environnement social (Arnould et al 2002, Boissy et al 2001b, Coutellier et al 2006, Roussel et al 2006, Valance et al 2006a, Veissier et al 2001, figure 3). La poursuite de ces travaux permettra d'apprécier les conséquences émotionnelles des perturbations de l'environnement social en fonction de l'espèce animale. Ces travaux devraient aider à l'élaboration de conduites d'élevage assurant une meilleure adéquation entre les besoins de l'animal et son environnement.

Par ailleurs, une approche neurobiologique a été amorcée afin d'identifier chez nos espèces animales les structures cérébrales impliquées dans la genèse des émotions. Certaines régions du cortex, notamment les aires préfrontales, jouent un rôle essentiel dans la représentation mentale des émotions et donc des états de bien-être (Rolls 2005). Ces données, obtenues essentiellement chez les primates, sont difficilement transposables aux espèces d'intérêt agronomique, notamment pour des problèmes d'homologie de structures, même si certaines de ces structures font désormais l'objet d'étude en neuroanatomie fonctionnelle

Figure 2. Mesure du nombre de sursauts et de la fréquence cardiaque d'agnelles exposées à l'apparition soudaine d'un objet précédée d'un signal lumineux (signalé) ou non (aléatoire).

\section{Sursauts}

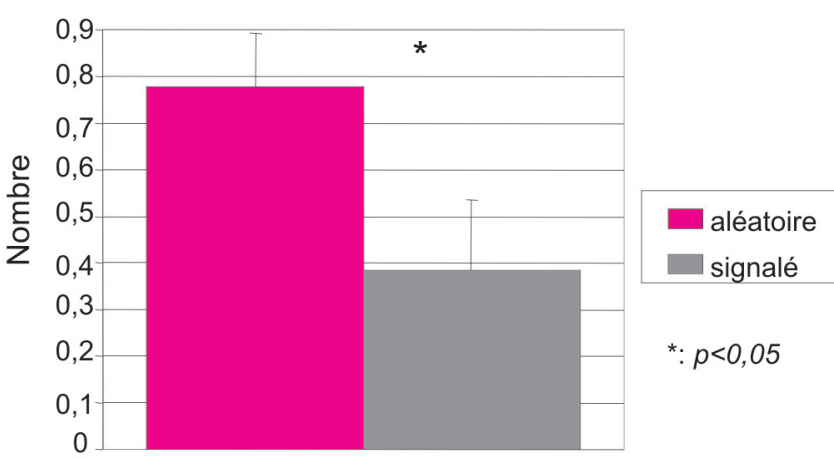

\section{Fréquence cardiaque}

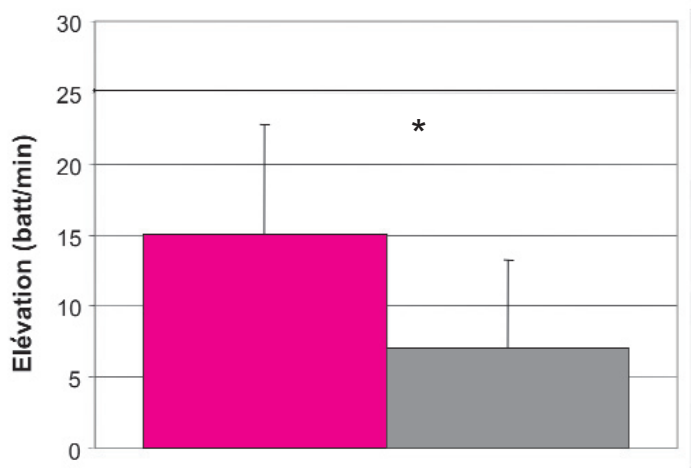

Les réactions spécifiques de la soudaineté (sursaut et tachycardie) sont plus faibles chez les agnelles pour lesquelles l'événement soudain est signalé et donc prévisible. 
Figure 3. Mesure de l'activité locomotrice de porcelets (46 jours d'âge) exposés à un événement soudain (jet d'eau). Les porcelets avaient été préalablement sevrés à différents âges (1, 3 et 4 semaines, respectivement W7, W21 et W28) ou maintenus avec leur mère $(C)$.

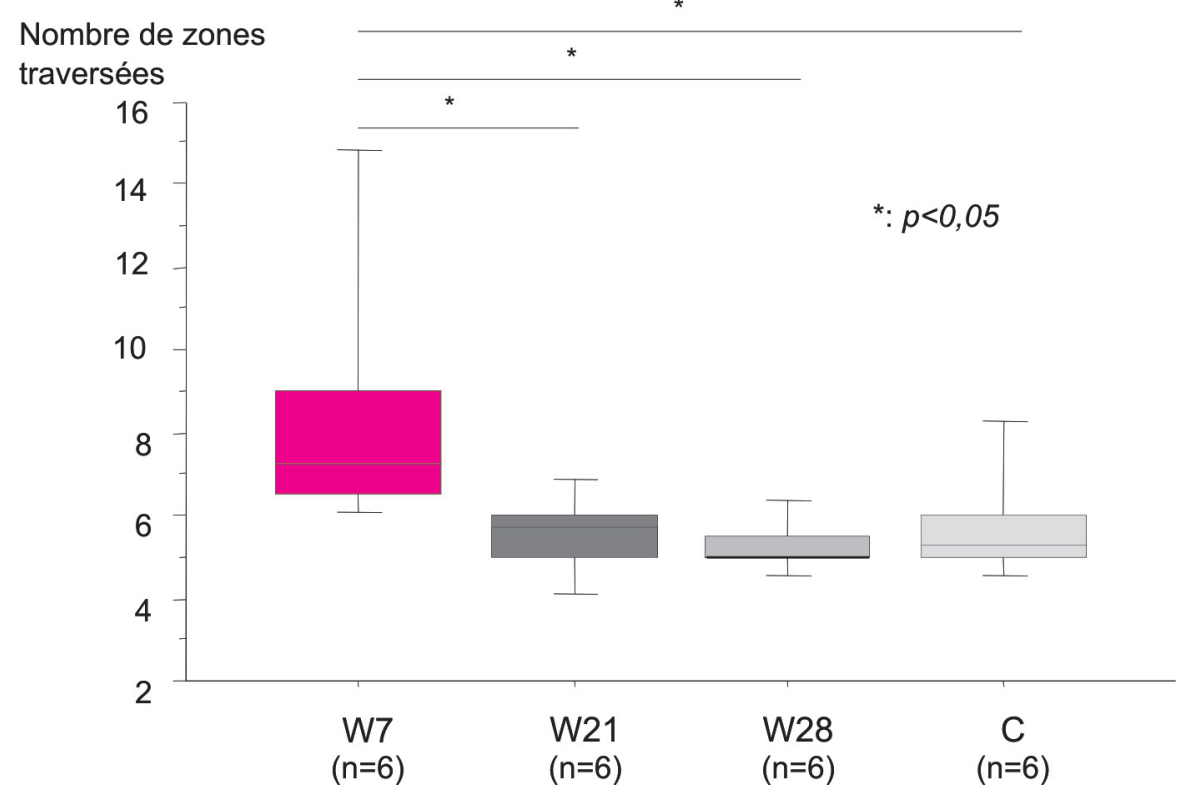

Les porcelets sevrés le plus précocement (W7) sont les plus réactifs à la soudaineté. chez l'ovin (Keller et al 2005). Quoi qu'il en soit, il est clair que ce sont des structures sous-corticales, entre autres les noyaux amygdaliens, qui sont à la base des émotions telles que la colère et la peur (Panksepp 1998). Nous avons donc initié une approche comparative sur ovins et cailles (figure 4), avec une attention particulière pour l'amygdale et son homologue chez l'oiseau. Les nouvelles techniques d'imagerie cérébrale, utilisables sur animaux vigiles avec une résolution satisfaisante, permettent d'envisager de nouveaux développements dans le domaine de l'exploration des mécanismes neurobiologiques des émotions chez les animaux d'élevage.

\section{2 / Des émotions dans la cognition ou comment comprendre la mise en place d'états affectifs persistants}

Si les processus cognitifs sont à l'origine des émotions, les émotions peuvent en retour influencer les processus cognitifs. De nombreux travaux en psychologie humaine montrent combien le vécu émotionnel peut biaiser la manière dont l'individu traite les informations provenant de son environnement. Cela concerne à la fois des altérations de l'attention, de la mémoire événement ambigu (Wright et Bower 1992). De telles modifications cognitives consécutives à une émotion existent également chez l'animal (Paul et al 2005). Ainsi, des génisses fortement stressées sont incapables d'abandonner un comportement préalablement appris qui n'est plus récompensé, ce qui les empêche d'acquérir un nouveau comportement plus approprié (Lensink et al 2006). Par contre, un stress plus modéré facilite les processus d'apprentissage : après avoir reçu une injection de catécholamines mimant la composante physiologique d'une émotion modérée, les rats sont plus attentifs et améliorent leurs performances mnésiques (Sandi et al 1997). Des phénomènes similaires ont été décrits chez le poussin (Gibbs et Summers 2000).

La mise en évidence de répercussions immédiates d'une émotion sur les processus cognitifs laisse entrevoir l'intérêt de développer un nouvel axe de recherche. Il s'agit d'explorer comment l'accumulation d'émotions peut modifier de manière durable les fonctions cognitives de l'animal et par voie de conséquence instaurer chez ce dernier un état persistant de bien-être ou au contraire de mal-être. Il existe déjà quelques travaux sur les animaux de laboratoire qui suggèrent combien une expérience émotionnelle peut moduler durablement les capacités d'évaluation des animaux. Par exemple, des rats ou des souris soumis de manière répétée à des événements imprévisibles, connus pour induire un état de stress, présentent une altération de leurs capacités à juger et à prendre des décisions (respectivement : Harding et al 2004, à interpréter négativement tout nouvel

Figure 4. Mesure de l'activation neuronale par immunohistochimie (protéine Fos) dans le noyau paraventriculaire de l'hypothalamus sur des cailles exposées ou non avant sacrifice à un environnement nouveau.

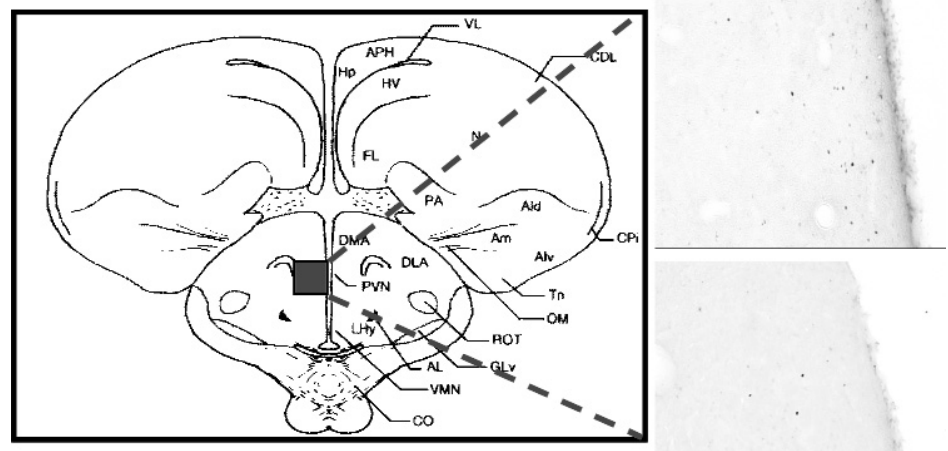

Caille non stimulée 
Pardon et al 2000). La mise en évidence de tels biais systématiques dans la manière dont l'animal évalue son environnement, pourrait alors permettre de les utiliser comme autant d'indicateurs d'un état d'inconfort. Par ailleurs, il a été montré qu'un animal qui est capable de contrôler son environnement par un comportement appris, apparaît par la suite moins perturbé par de nouvelles contraintes qu'un animal ne pouvant maîtriser son environnement car imprévisible ou incontrôlable (Dantzer 1989). La mise en évidence de critères élémentaires d'évaluation que l'animal est amené à utiliser de manière rémanente en raison des particularités de l'environnement, devrait permettre de comprendre pourquoi un stress chronique conduit dans certains cas à une apathie ou un émoussement affectif et dans d'autres cas à une exacerbation de la réactivité émotionnelle. Une apathie se développerait lorsque l'animal n'a aucun moyen d'action sur les événe- ments négatifs, alors qu'une hyperréactivité résulterait du fait que l'animal soit parvenu à les contrôler.

\section{Conclusion}

L'étude des interactions entre émotions et cognition représente une démarche innovante pour approcher de manière rigoureuse chez l'animal la nature de ses états affectifs. C'est une approche intégrative pluridisciplinaire (comportement, psychologie, physiologie, génétique et neurobiologie) que nous nous efforçons de développer sur les animaux en élevage. La poursuite de l'identification des critères d'évaluation, définis selon le cadre conceptuel inspiré des travaux en psychologie cognitive, permettra de mettre en place une véritable étude comparative à la fois entre espèces (les espèces utilisées en élevage ayant des capacités cogniti- ves plus ou moins développées) et intra espèce (les capacités cognitives évoluant avec l'âge et variant selon la race et le sexe) dans le but à terme de préciser les conditions de bien-être en relation avec les capacités cognitives des animaux. Une fois les critères d'évaluation identifiés, l'étude de leur agrégation facilitera l'identification à la fois du répertoire émotionnel de nos animaux et des caractéristiques génératrices d'inconfort ou de confort en élevage. Par ailleurs, l'analyse des biais cognitifs sous l'effet d'émotions répétées permettra de mieux comprendre la mise en place d'états affectifs persistants de bien-être/mal-être. En particulier, il s'agira d'identifier les biais cognitifs acquis et/ou les prédispositions cognitives susceptibles de générer des désordres affectifs durables à l'origine des états de mal-être.

\section{Références}

Arnould C., Quekenborn D., Mills A.D., Faure J.M., 2002. Behaviour and emotional reactivity of quails reared in stable or unstable groups. $36^{\text {th }}$ Int. Cong. Int. Soc. Appl. Ethol., Wageningen, The Netherlands, 170

Bradley B.P., Mogg K., Lee S.C., 1997. Attentional biases for negative information in induced and naturally occurring dysphoria. Behav. Res. Therapies, 35, 911-927.

Boissy A., Nowak R., Orgeur P., Veissier I., 2001a. Les liens sociaux chez les ruminants d'élevage : limites et moyens d'action pour favoriser l'intégration de l'animal dans son milieu. INRA Prod. Anim., 14, 79-90.

Boissy A., Veissier I., Roussel S., 2001b. Emotional reactivity affected by chronic stress: an experimental approach in calves subjected to environmental instability. Anim. Welf., 10, S175-S185.

Coutellier L., Arnould C., Boissy A., Orgeur P., Prunier A., Veissier I., Salaun M. C., 2006. Pig's responses to repeated social regrouping and relocation during the growing-finishing period. Applied Anim. Behav. Sci., sous presse.

Dantzer R., 1989. L'illusion psychosomatique. Editions Odile Jacob, Paris, France, 121p.

Dantzer R., 2002. Can farm animal welfare be understood without taking into account the issues of emotion and cognition? J. Anim. Sci., 80, E1-E9.

Dawkins M.S., 1983. La souffrance animale. Editions du Point Vétérinaire, Maisons-Alfort, France, 152p.

Dawkins M.S., 2001. How can we recognise and assess good welfare? In: Coping with challenge: Welfare in animals including humans. Broom, D. M. (Ed.), Dahlem University Press, Berlin, Allemagne, 63-76.
Désiré L., Boissy A., Veissier I., 2002. Emotions in farm animals: a new approach to animal welfare in applied ethology. Behav. Proc., $60,165-180$

Désiré L., Veissier I., Després G., Boissy A., 2004. On the way to assess emotions in animals: Do lambs evaluate an event through its suddenness, novelty or unpredictability? J. Comp. Psychol., 118, 363-374.

Désiré L., Veissier I., Després G., Delval E., Toporenko G., Boissy A., 2006. Appraisal process in sheep: interactive effect of suddenness and unfamiliarity on cardiac and behavioural responses. J. Comp. Psychol., 120, 280-287.

Després G., Boissy A., Désiré L., Le Neindre, P. Veissier I., 2003. Validation of the measure of sympatho-vagal effect in lambs through autonomic blockades and heart rate variability indexes. J. Anim. Vet. Adv., 2, 615-619.

Duncan I.J.H., 1996. Animal welfare in terms of feelings. Acta Agric. Scand., 27, 29-35.

Gibbs M.E., Summers R.J., 2000. Separate roles for $\beta_{2}$ - and $\beta_{3}$-adrenoceptors in memory consolidation. Neuroscience, 95, 913-922.

Greiveldinger L., Veissier I., Boissy A., 2006. Predictability and expectations influence emotional responses in lambs. In: Quality of life symposium, London, UK, 13-14 September, 93.

Harding E.J., Paul E.S., Mendl M., 2004. Cognitive bias and affective state. Nature, 427, 312

Keller M., Meurisse M., Levy F., 2005. Mapping of brain networks involved in consolidation of lamb recognition memory. Neuroscience, 133, 359-369.

Lazarus R.S., 1991. Progress on a cognitivemotivational-relational theory of emotion. Am. Psychol., 46, 819-834

Lensink B.J., Veissier I., Boissy A., 2006. Enhancement of performances in a learning task in suckler calves after weaning and relocation: Motivational versus cognitive control? A pilot study. Applied Anim. Behav. Sci., 100, 171-181.

Mason J.W., 1971. A re-evaluation of the concept of "non-specificity" in stress theory. J. Psych. Res., 8, 323-333.

Mendl M., Burman O., Laughlin K., Paul E., 2001. Animal memory and animal welfare Anim. Welf., 10, S17-S25.

Mendl M., Paul, E.S., 2004. Consciousness, emotion and animal welfare: insights from cognitive science. Anim. Welf., 13, S17-S25.

Panksepp J. 1998. Affective neuroscience: The foundations of human and animal emotions. Oxford University Press, New-York, USA, 466p.

Pardon M.C., Perez-Diaz F., Joubert C., Cohen-Salmon C., 2000. Influence of a chronic ultramild stress procedure on decision-making in mice. J. Psych. Neurosci., 25, 167-177.

Paul E.S., Harding E.J., Mendl M., 2005. Measuring emotional processes in animals: the utility of a cognitive approach. Neurosci. Biobehav. Rev., 29, 469-491.

Reisberg D., Heuer F., 1995. Emotion's multiple effects on memory. In: Brain and memory: modulation and mediation of neuroplasticity. Mc Gaugh J.L., Weiberger N.M., Lynch G. (Eds.), Oxford University Press, Oxford, UK, 84-92.

Rolls E.T., 2005. Emotion explained. Oxford University Press, Oxford, UK, 606p.

Roussel S., Boissy A., Montigny D., Hemsworth P.H., Duvaux-Ponter C., 2005. Gender-specific effects of prenatal stress on emotional reactivity and stress physiology of goat kids. Horm. Behav., 47, 256-266.

Roussel S., Hemsworth P.H., Leruste H., White C., Duvaux-Ponter C., Nowak R., Boissy A., 2006. Repeated transport and isolation during pregnancy in ewes: effects on the reactivity 
to humans and to their offspring after lambing. Applied Anim. Behav. Sci., 97, 172-189.

Sandi C., Loscertales M., Guaza C., 1997. Experience-dependent facilitating effect of corticosterone on spatial memory formation in the water maze. Eur. J. Neurosci., 9, 637-642.

Scherer K. R., 1987. Toward a dynamic theory of emotion: the component process model of affective states. Geneva Studies in Emotion and Communication, 1, 1-98.

Scherer K. R., 1999. Appraisal theories. In: Handbook of cognition and emotion, $T$.
Dalgleish, M. Power (Eds), Wiley, Chichester, UK, 637-663.

Valance D., Boissy A., Despres G., Constantin P., Leterrier C., 2007a. Emotional reactivity modulates autonomic responses to an acoustic challenge in quail. Physiol. Behav., 90, 165-171.

Valance D., Desprès G., Boissy A., MignonGrasteau S., Constantin P., Leterrier C., 2007b. Genetic selection on a behavioral fear trait is associated with changes in heart rate variability in quail. Genet. Brain Behav., sous presse.
Valance D., Leterrier C., Després G., Galand C., Favreau A., Boissy A., 2006. Behavioural and cardiac responses to changes in the social and non social environment in quail. 40 $\mathrm{th}$ Int Cong. Int. Soc. Appl. Ethol., Bristol, UK, 190.

Veissier I., Boissy A., de Passillé A.M., Rushen J., van Reenen C.G., Roussel S., Andanson S., Pradel P., 2001. Calves' responses to repeated social regrouping and relocation. J. Anim. Sci., 79, 2580-2593.

Wright W.F., Bower G.H., 1992. Mood effects on subjective probability assessment. Org. Behav. Human Decision Processes, 52, 276-291.

\section{Résumé}

La notion de bien-être chez l'animal d'élevage n'a de sens que si on lui reconnaît la capacité à ressentir des émotions. Cependant, du fait de l'absence de langage verbal, le vécu émotionnel de l'animal est difficilement mesurable, ce qui a toujours rendu son étude délicate. Les travaux récents en psychologie cognitive montrent combien l'étude des interactions entre émotions et cognition est nécessaire pour accéder aux états affectifs de l'animal et tenter ainsi d'identifier les paramètres susceptibles d'affecter son bien-être. Ce texte comporte deux volets. Le premier volet s'appuie sur les théories de l'évaluation développées chez l'Homme, lesquelles suggèrent que le déclenchement des émotions résulte de processus cognitifs d'évaluation basés sur des critères élémentaires tels que la nouveauté et la prévisibilité. Sur la base de ce cadre théorique, nous avons engagé une approche sur les animaux d'élevage visant à définir les critères d'évaluation qu'ils utilisent, et à faciliter ainsi la compréhension de leurs émotions à partir de l'étude de la combinaison des critères impliqués. Le second volet de ce texte s'appuie sur les nombreuses études menées chez l'Homme et les animaux de laboratoire, qui suggèrent que les émotions peuvent en retour influencer les capacités cognitives de l'individu en altérant ses capacités attentionnelles et mnésiques. L'approfondissement de l'étude des interactions entre émotions et cognition offre donc de nouvelles perspectives de recherche pour mieux comprendre les conditions de bien-être des animaux en élevage.

\section{Abstract}

\section{Emotion and cognition: a new strategy to achieve animal welfare}

Assessment of farm animal welfare requires a good understanding of the animals' affective experience, including their emotions. However, this is difficult to measure because of the absence of verbal communication. Recent studies in the field of cognitive psychology have shown that affective states can be assessed through investigation of the interactions between emotions and cognition. On the one hand, theories of the way humans appraise situations provide a conceptual framework that suggests that emotions are triggered by cognitive appraisal processes based on few elementary criteria such as novelty and predictability. In accordance with appraisal theories, we have developed an experimental approach to study the elementary criteria used by farm animals to evaluate their environment and then express a given emotional response. On the other hand, an increasing number of studies in humans and animals suggest that emotions also influence cognitive processes by modifying attention, memory and judgement. Measurement and manipulation of cognitive processes may provide new insights into how emotions and more persistent affective states in animals can be assessed. Further work on these cognitive approaches will offer new paradigms for a better understanding of the welfare of farm animals.

BOISSY A., ARNOULD C., CHAILlOU E., COLSON V., DÉSIRÉ L., DUVAUX-PONTER C., GREIVELDINGER L., LETERRIER C., RICHARD S., ROUSSEL S., SAINT-DIZIER H., MEUNIER-SALAÜN M.C., VALANCE D., 2007. Emotions et cognition : stratégie pour répondre à la question de la sensibilité des animaux. INRA Prod. Anim., 20, 17-22. 
